\title{
La Inteligencia Intrapersonal en el aula de ELE: una propuesta didáctica para alumnos sinohablantes
}

\author{
MARTA MARTÍNEZ RUBER \\ Universidad de Zaragoza \\ mruber@unizar.es
}

\begin{abstract}
Resumen: El proceso de adquisición del español como lengua meta de los estudiantes sinohablantes se ve dificultado por el elevado índice de variables conflictivas de índole lingüística, emocional y cultural a las que deben hacer frente durante el período de aprendizaje de la L2. De todos, los aspectos relativos a la variable afectiva son los que cuentan en la actualidad con menos atención y propuestas metodológicas concretas para aplicar en el trabajo de aula. La unidad didáctica que aquí se presenta está pensada para trabajar las necesidades de los alumnos chinos en el ámbito de la inteligencia intrapersonal y se ocupa de las variables afectivas autorreferenciales de la autoestima y la motivación personal. En el artículo se desarrolla la unidad didáctica atendiendo a su estructura y objetivos psicoemocionales y, finalmente, se comentan los beneficios tras una aplicación preliminar e informal entre los alumnos a los que iba destinada.
\end{abstract}

Palabras clave: estudiante sinohablante, inteligencia intrapersonal, choque cultural, método comunicativo.

Intrapersonal intelligence in the classroom of Spanish as a Foreign Language: a didactic proposal for Chinese students

\begin{abstract}
The acquisition process of Spanish as a target language for Chinese students is hindered by the variety and complexity of linguistic, emotional and cultural issues they must face over the course of their second language acquisition process. Above all, the emotional aspects are the ones which currently attract less attention and fewer specific methodological proposals that can be put into practice in the classroom. The didactic unit hereby presented has been conceived in order to work for the needs of Chinese students within the aspect of their intrapersonal intelligence and also involves the two introspective emotional variables of self-esteem and personal motivation. This paper shows the development of a didactic unit focusing on its psychoemotional structure and objectives and lastly, the benefits that result from a preliminary and informal execution in a classroom with Chinese students.
\end{abstract}

Key words: Chinese student, intrapersonal intelligence, cultural shock, communicative approach.

\section{Introducción}

La multiplicidad de factores culturales y cognitivos que obstaculizan el aprendizaje de E/LE de los estudiantes sinohablantes se está viendo agravada por la falta de respuestas metodológicas y didácticas que trabajen con efectividad la especial idiosincrasia de este alumnado. Como resultado, encontramos una ralentización generalizada en su proceso de 
adquisición lingüística $\mathrm{y}$, aunque en el momento actual las investigaciones han logrado clasificar las causas de dichos obstáculos (Couto, 2004; Lu, 2009), es evidente la ausencia de materiales pedagógicos para el aula.

Por otro lado, a pesar de que el análisis de necesidades ha puesto de manifiesto que las dificultades de aprendizaje de los estudiantes sinohablantes atañen tanto a la variable cognitiva como a la afectiva, lo cierto es que pocos estudios han incidido en la importancia de trabajar la inteligencia intrapersonal como medio de acompañar a nuestros alumnos chinos en su proceso de adquisición lingüística.

Enfocada en la parte más íntima del ser, la inteligencia intrapersonal se encarga de anclarnos al entorno. Es responsable de poner en marcha el proceso de aprendizaje y de mantenerlo constante a lo largo del tiempo con independencia de los escollos que encontremos en el camino (Gardner, 1983). Al ocuparse de las variables de autoestima y motivación, una falla en la inteligencia intrapersonal afectará, por tanto, más profundamente a la adquisición lingüística que si dichas limitaciones se dan en cualquier otra de las inteligencias múltiples (Rubio Alcalá, 2002).

Sin embargo, el estudiante sinohablante en situación de inmersión es especialmente vulnerable a sufrir una devaluación en su autoimagen. La aplicación del método comunicativo con estos aprendientes ha evidenciado la existencia de elementos de conflictividad entre este enfoque metodológico y sus creencias y actitudes previas de aprendizaje. Al provenir de una cultura educativa en la que se fomentan valores como la receptividad, la competitividad y el individualismo, ¿se encuentran estos alumnos realmente preparados para integrarse en un sistema de enseñanza holístico que trabaja con las dimensiones cognitiva, emotiva y volitiva del estudiante?

Para responder a esta pregunta hay que entender que el método comunicativo no supone para estos discentes una simple modificación de aspectos superficiales de la dinámica del aula, sino que implica adaptarse a un cambio de paradigma en lo que a la concepción de la adquisición lingüística y al rol del estudiante se refiere. Educados en un sistema educativo de corte tradicional asentado sobre las bases filosóficas del confucianismo (Sánchez Griñán, 2009), el alumno sinohablante en situación de inmersión deberá pasar de ejercer un rol receptivo a un rol activo, de orientarse según una motivación extrínseca a una intrínseca y de ver la lengua ya no como un conjunto de hábitos susceptibles de ser adquiridos, sino como un sistema articulado de intercambios comunicativos complejos.

Sin embargo, acostumbrados a usar casi en exclusividad la memoria como medio para la adquisición lingüística de la L2, la necesidad de incorporar al aprendizaje procesos cognitivos complejos como el análisis, la síntesis, la inducción y la deducción y de tener que desarrollar estrategias cognitivas, metacognitivas y afectivas como forma de convertirse en buenos aprendientes de lenguas, hace que el alumno sinohablante en situación de inmersión viva un choque cultural muy intenso (Gibert y Colón, 2014).

Ante este hecho, no resulta extraño que el alumno chino pueda ver comprometida su autoestima. Afrontar cambios tan nucleares en su identidad como aprendiente facilita la aparición de emociones negativas y un sentido de inadecuación personal que lo alejan del aprendizaje productivo. Sin embargo, un sistema educativo tan individualista y competitivo como el chino (Galloso Camacho, 2014) no favorece el buen desarrollo de la inteligencia intrapersonal. Antes bien, generará aprendientes carentes de una autoimagen 
sólida y, por lo tanto, con dificultades para solventar los problemas propios de un aprendizaje comunicativo.

\section{Causas de la dificultad de los estudiantes sinohablantes para la adquisición del español como LM y sus efectos en la variable afectiva}

Las dificultades que los estudiantes sinohablantes encuentran durante su proceso de adquisición lingüística no radica tanto en lo específico de las causas, pues todas ellas las encontramos en otros grupos étnico-lingüísticos, como en la multiplicidad e imbricación de los factores en juego.

Mientras estudiantes de otros grupos lingüísticos y culturales presentan dificultades específicas en alguna de las áreas del aprendizaje, los alumnos sinohablantes muestran dificultades significativas en los tres ámbitos relacionados con este: el ámbito lingüístico, el ámbito sociocultural y el ámbito educativo.

De igual manera, la distancia que existe entre docente y discentes es tan dilatada que, de no encontrarse este preparado para afrontarla, repercutirá en el aula negativamente. Si el docente ignora las claves para entender el bagaje cultural de nuestros alumnos chinos, así como su postura ante el proceso de enseñanza-aprendizaje, «puede crearse un conflicto que perjudicará el objetivo que ambos comparten: el aprendizaje de la lengua» (Gibert y Gutiérrez-Colón 2014: 93).

Visto el elevado número de alumnos sinohablantes que presentan problemas de fosilización lingüística, actitudinales y de absentismo, parece lógico afirmar que la dificultad específica de nuestros alumnos sinohablantes se debe a estas causas: la imbricación de dificultades lingüísticas y emocionales provocadas por el choque cultural, y el hecho de que los docentes occidentales no nos encontramos siempre bien preparados para impartir clases a este colectivo.

\subsection{La distancia tipológica entre ambas lenguas}

Lu (2015:70-71) sintetiza las dificultades lingüísticas que los hablantes de chino experimentan durante el aprendizaje del español. Abarcan todos los niveles del idioma y su origen e importancia radica, fundamentalmente, en la enorme distancia tipológica existente entre ambas lenguas. El paso de una lengua aislante como el chino a una lengua flexiva como el español origina para nuestros alumnos dificultades en los sistemas morfológico, gramatical y sintáctico. De igual manera, el paso de una lengua tonal a una lengua no tonal provoca no pocas dificultades a nivel fonético.

En el sistema fonético, grosso modo, los estudiantes sinohablantes presentarían

[...] problemas con la pronunciación de los fonemas vibrantes e interdentales que no existen en el chino y los fonemas oclusivos en parejas sorda-sonora que aparecen en chino todas sordas y en parejas aspirada no aspirada; la distinción entre los dos vibrantes y entre éstos y el lateral; la estructura silábica española mucho más complicada que la china; los grupos consonánticos, sobre todo, aquellos formados con uno de los fonemas vibrante y lateral. (Lu, 2015: 70)

En lo referente al resto de los sistemas lingüísticos, el manejo del plano morfológico supondrá un gran esfuerzo para el alumno chino pues, acostumbrados a la ausencia de morfología nominal y verbal, la adquisición del español les exigirá interiorizar 
variaciones de «género y número para sustantivos, pronombres, adjetivos y artículos, caso para pronombres personales, modo, tiempo y persona para verbos» (Lu, 2015: 70).

Si nos centramos en la estructura sintáctica, esta resulta especialmente problemática para nuestros alumnos. Mientras que las oraciones españolas se estructuran alrededor de relaciones funcionales explícitas (las secuencias sintácticas de sujeto y predicado), las oraciones del chino lo hacen en torno a funciones lógicas implícitas (las nociones de tópico/ comento). Por lo tanto, en la oración china «las relaciones gramaticales entre las ideas dependen en gran medida del orden de palabras y del contexto en que se encuentran» ( $\mathrm{Lu}, 2015:$ 71), mientras que en la oración española se construye a partir de la segmentación de unidades sintácticas en constituyentes jerarquizados.

Esta distancia tipológica multiplicará la propensión al error de los aprendientes sinohablantes, especialmente durante los niveles iniciales. Factor que acabará incidiendo negativamente en la variable afectiva, pues, como afirma Couto (2014: 124), los hablantes chinos de niveles iniciales (A1 y A2) presentan una mayor propensión a cometer errores haciendo que su ritmo de aprendizaje sea más lento, lo que provoca ansiedad y frustración en ellos.

\subsection{El choque cultural: distancia sociocultural y efectos en el alumno}

La distancia cultural entre China y España es grande. Herederas de una tradición filosófica e histórica de muy variada raigambre, ambas culturas se filtran en el aula de E/LE dificultando la comunicación entre los estudiantes y el profesor y provocando un choque cultural que se opone al buen fluir del proceso de enseñanza-aprendizaje.

Lo primero que habrá que tener en cuenta es que nuestros alumnos chinos provienen de una cultura colectivista y fuertemente jerarquizada. Para los chinos, por lo tanto, «no identificarse con un colectivo es negativo y a quien se aparta del grupo se lo puede llegar a tratar con indiferencia e incluso desprecio» (Gibert y Gutiérrez-Colón, 2014: 97). Esta relación entre sociedad e individuo provocará inevitablemente cortocircuitos en el aula comunicativa, pues tomar la iniciativa en la palabra será también considerado abandonar el grupo si el estudiante no ha buscado antes la aprobación de sus iguales.

Grupo primigenio de pertenencia, el alumno sinohablante aprende en la familia una forma de relacionarse organizada alrededor de los conceptos de autoridad, magisterio y obediencia. Conceptos que el alumno reproducirá en el aula no solo por ser el parámetro de pensamiento que estructura sus relaciones con el otro, sino por el peso específico que la familia tiene en la cultura china. Los alumnos chinos establecen una fuerte proyección de la figura paterna sobre la del profesor del que esperan, tal y como afirman Gibert y Gutiérrez Colón (2014: 94), un dominio completo sobre su materia de enseñanza. Y, también, que se maneje como ejemplo de autoridad dentro del aula y modelo a seguir fuera de ella.

Las consecuencias del choque cultural en relación con los roles profesor-alumno son elevadas, por lo tanto, en términos de interacción. Mientras el profesor de E/LE de un aula comunicativa espera de sus alumnos una retroalimentación activa y una interacción fluida, los estudiantes sinohablantes guardarán silencio por temor a socavar la autoridad del discente (Gibert y Gutiérrez-Colón, 2014: 95). Para ellos, preguntar a quien tiene la 
autoridad se considera una falta de respeto y, si le sumamos el miedo a dar una imagen demasiado individualista, la inhibición de los estudiantes será todavía mayor.

En esta misma línea, dado que los alumnos asocian la figura del profesor a la del padre, es común que estos mantengan contacto con el docente fuera del aula a través de aplicaciones telefónicas (Vázquez Torronteras, 2018: 8), actitud que hemos podido comprobar en nuestros alumnos sinohablantes en situación de inmersión. Por lo tanto, en el caso de que el docente español se desvincule de los alumnos al terminar la jornada laboral, estos pueden sentir, sobre todo al estar en un país extranjero, una sensación de desamparo y abandono que acentúe sentimientos de inadecuación y dificulte todavía más la interacción en el aula.

Las dinámicas de autoridad dentro de la familia provocan también que todavía en la actualidad haya casos en los que «[...] el hijo siga los mandatos del padre en cuanto a educación, llegando a estudiar una carrera universitaria por premisa de su padre, lo que propicia graves casos de desmotivación en el aula» (Vázquez Torronteras, 2018: 5). Si, además, tenemos en cuenta, como afirma Lu (2015: 66), la revalorización de los estudios de filología hispánica en China desde el año 2000 debido a las buenas relaciones económicas entre el gigante asiático y los países hispanohablantes, no debe resultarnos extraño encontrar en nuestras aulas a algunos alumnos que presenten una baja motivación.

\subsection{El choque cultural: sistemas educativos divergentes y efectos en el aprendizaje}

Educados en un sistema de enseñanza de corte confucianista, los estudiantes chinos están acostumbrados a recibir una educación memorística y fuertemente competitiva. Esta competitividad es tal que los niños son segregados desde edades muy tempranas con el objetivo de conseguir plaza en las mejores universidades (Galloso Camacho, 2014: 2). Situación que tendrá por consecuencia la llegada a nuestras aulas de buenos alumnos marcados por historias de fracaso académico y, en consecuencia, con una baja autoimagen que afectará negativamente a la variable afectiva del aprendizaje.

Quizá la característica más sobresaliente de la aplicación del confucianismo a la educación sea la focalización en el aprendizaje memorístico. Es cierto que en el confucianismo la memorización no tiene el mismo significado que en el conductismo. El confucianismo pretende que a través de la práctica memorística los alumnos sinohablantes sean capaces de trascender el aprendizaje para adentrarse en terrenos que les harán aprehender los contenidos en su sentido último (Sánchez Griñán, 2009: 4).

No obstante, el preeminente lugar de la memoria en la educación china tiene por consecuencia que el sistema ponga su foco en la realización de pruebas antes que en la adquisición de conocimientos. Como resultado, los discentes estudian antes para superar exámenes que para aprender y no están acostumbrados a activar la motivación intrínseca, responsable del aprendizaje significativo. Encontramos así otra de las causas de los problemas emocionales y actitudinales de los alumnos sinohablantes. Para tener éxito en un entorno de aprendizaje comunicativo en el que se usa una evaluación continua y formativa, el alumno necesitará haber desarrollado una sólida motivación intrínseca, es decir, grandes dosis de responsabilidad personal y de autorregulación en lo relativo al proceso de enseñanza-aprendizaje. 


\subsection{Limitaciones de los estudiantes sinohablantes a la hora de afrontar el aprendizaje de E/LE según el método comunicativo}

Las características socioculturales y educativas analizadas trazan un perfil de alumno que se encuentra bastante alejado del llamado buen alumno o aprendiente de lenguas, entendiendo por buenos aprendientes «[...] aquellos que son eficaces, activos y que, simplemente saben cómo aprender» (Giovannini et al., 1996: 24).

Para manejarse con éxito en el enfoque comunicativo los estudiantes deben adoptar un rol activo y autónomo, ser capaces de responsabilizarse de su propio proceso de aprendizaje y orientar la voluntad a la consecución de objetivos comunicativos concretos. Sin embargo, la fuerte tendencia memorística de los alumnos sinohablantes afecta directamente su capacidad crítica. Potentes en la aplicación de estrategias memorísticas, carecen, sin embargo, de la capacidad de emitir juicios críticos, de habilidades investigadores y de la aptitud para saber dónde se encuentran en relación con el propio proceso de adquisición lingüística.

La pobreza en la aplicación de estrategias cognitivas, metacognitivas, comunicativas y socioafectivas se agrava por la abrumadora confianza de estos estudiantes en la memoria, que llega hasta el punto de incapacitarlos para aceptar, tal y como explica Sánchez Griñán, otro enfoque de enseñanza diferente al conocido. En parte, quizá, porque tampoco son capaces de comprender cuáles son los objetivos que el profesor persigue dentro de ese nuevo enfoque de enseñanza (Bega González, 2015: 229).

Debido a que saber elegir las estrategias de aprendizaje adecuadas está íntimamente ligado con el éxito del aprendizaje, las consecuencias de desarrollar «un aprendizaje memorístico, sin aplicaciones contextuales ni posibilidad de razonamiento» (Bega González, 2015: 236-237), derivan en la incapacidad del estudiante para aprehender los principios del razonamiento en español, la lógica inherente al idioma.

Esta falta de razonamiento entronca con un concepto de error definido también en términos negativos. En el sistema educativo chino el error es penalizado y, por lo tanto, percibido por los estudiantes como una falta que debe ser reparada inmediatamente. Se asocia así de forma directa a sentimientos de inadecuación y de vergüenza, por lo que será muy difícil para nuestros alumnos afrontarlo sin una merma en su autoestima.

Este factor repercute de forma directa en la capacidad oral de los estudiantes. Al tener miedo a equivocarse, se inhiben reduciendo considerablemente las oportunidades comunicativas. Y, cuando se animan a hablar, una excesiva atención a la forma y un exceso de monitorización impide el estado de relajación necesario que fomenta la creatividad. «Después de haber cometido un error, tienen menos seguridad y, por lo tanto, también menos ganas de volver a hablar» (Chien, 2008: 39).

Teniendo en cuenta la globalidad de los factores hasta aquí enunciados ¿cómo se sentirá en el aula de E/LE un alumno para el que conceptos como uso comunicativo de la lengua, creatividad o utilidad del error son desconocidos? ¿Qué ocurrirá con el alumno sinohablante cuando entre a formar parte de un sistema educativo que otorga a la memorización un papel subsidiario y fomenta, en su lugar, habilidades de alto rango cognitivo como la interpretación, el procesamiento y la selección de la información? ¿Será capaz de responsabilizarse de su propio proceso de aprendizaje y asumir un papel diametralmente opuesto a aquel al que está acostumbrado? 
Parece lógico pensar que el alumno sinohablante pueda generar ciertos sentimientos de desubicación, falta de autoestima e inhibición que en nada favorezcan el aprendizaje exitoso de la L2.

\section{5. ¿Por qué es tan importante trabajar la inteligencia intrapersonal con alumnos sinohablantes?}

Los problemas hasta aquí enunciados repercuten negativamente en la variable afectiva y, en concreto, en la inteligencia intrapersonal. Cuando Gardner (1983) reformuló la definición de inteligencia incorporando a sus cualidades la capacidad para resolver problemas, amplió el ámbito de la cognición humana y contribuyó a superar la idea de que la inteligencia se encuentra desvinculada del ámbito emocional. Con las aportaciones de Gardner (1983), Salovey y Mayer (1990) y Goleman (1995) entre otros, la inteligencia emocional recupera en lugar que le corresponde en el proceso de aprendizaje.

Según Goleman, la inteligencia intrapersonal es el conjunto de habilidades que nos permite $«[\ldots]$ configurar una imagen exacta y verdadera de nosotros mismos y que nos hace capaces de utilizar esa imagen para actuar en la vida de un modo más eficaz» (Goleman, 1995: 30).

Debido a que su finalidad última es la de producir mejores resultados en las acciones que emprendemos, un déficit en esta inteligencia entorpecerá e, incluso, podrá imposibilitar la adquisición del conocimiento. Este hecho no es nada que no hayamos comprobado en el aula, donde no resulta difícil darse cuenta de que «[...] los estudiantes que se hallan atrapados por el enojo, la ansiedad o la depresión tienen dificultades para aprender porque no perciben adecuadamente la información y, en consecuencia, no pueden procesarla correctamente» (Goleman, 1995: 8).

Un estado emocional negativo motivará, por lo tanto, una disminución en el rendimiento del alumno en un grado que puede variar desde leve o moderado hasta tan intenso que provoque el cierre cognitivo. En esta última situación el estudiante ya no es capaz de retener y procesar la información nueva porque toda su atención se encuentra absorbida por las emociones negativas (Goleman, 1995: 55).

Al producirse el cierre cognitivo, el estudiante ve afectada la memoria de trabajo y la capacidad de concentración $y$, dado que la memoria de trabajo «[...] es la función ejecutiva por excelencia de la vida mental, la que hace posible cualquier otra actividad intelectual, desde formular una frase hasta formular una compleja proposición lógica» (Goleman, 1995: 5), la adquisición lingüística resultará especialmente dañada.

Por otro lado, organizar un currículo basado en el enfoque comunicativo implica convertir al alumno en el centro del proceso de enseñanza-aprendizaje. Desde esta perspectiva se valora al discente en su dimensión holística y se trabajan de forma interdependiente las tres perspectivas del estudiante enunciadas en el Plan Curricular del Instituto Cervantes.

Considerarlo agente social, hablante intercultural y aprendiente autónomo significa capacitarlo para que pueda seleccionar los recursos lingüísticos que lo habiliten para desenvolverse con éxito en las transacciones comunicativas, para identificar los aspectos culturales más relevantes de la cultura de acogida y, por último, para responsabilizarse de su propio proceso de aprendizaje. 
Si quieren lograr el éxito en estos objetivos, los estudiantes deberán tener muy bien integradas la competencia existencial (saber ser) y la capacidad de aprender (saber aprender). Mientras la primera se ocupa de la importancia de los factores individuales en el proceso de aprendizaje: sus actitudes, sus motivaciones, sus estilos cognitivos y su tipo de personalidad, la segunda permite al alumno hacer un mejor uso de las oportunidades de aprendizaje con el objetivo de desarrollar las habilidades necesarias para que los nuevos conocimientos se integren con los ya existentes.

Por lo dicho hasta ahora, resulta evidente que los estudiantes sinohablantes presentarán importantes carencias en estas dos competencias, así como en las tres dimensiones anteriormente citadas. En resumen, nuestros alumnos sinohablantes tendrán problemas con las siguientes dimensiones del aprendizaje: incapacidad para asumir un rol activo y gestionar su propio proceso de aprendizaje, incapacidad para desarrollar el pensamiento crítico necesario para aprehender la nueva lengua, incapacidad para aplicar estrategias de aprendizaje destinadas a operar creativamente con la lengua y dificultades asociadas a la monitorización.

Los impedimentos que el alumno sinohablante encontrará en todos estos ámbitos afectarán invariablemente a su concepto del yo y, dado que la inteligencia intrapersonal es el filtro catalizador del resto de inteligencias, la capacidad que permite materializarse a todas las demás en el proceso de aprendizaje (Rubio Alcalá, 2002: 123), la importancia específica de esta inteligencia dentro de la variable efectiva parece evidente.

Las variables afectivas que hay que tener en cuenta a la hora de trabajar la inteligencia intrapersonal en el aula son, dentro de las emociones negativas, la ansiedad, la inhibición, la frustración y la tensión y dentro de las positivas: la autoestima, la valoración y el sentido del yo. Todas ellas relacionadas con la autoimagen y, en consecuencia, con la autoeficacia.

Si nos centramos en el ámbito del aprendizaje de segundas lenguas, la importancia de trabajar la inteligencia intrapersonal se incrementa. Como afirma Arnold (1999), la inteligencia intrapersonal cobra un peso específico en nuestra disciplina debido a que el aprendizaje de idiomas es un proceso especialmente sensible al estado emocional del alumno.

La ansiedad es posiblemente el factor afectivo que obstaculiza con mayor fuerza el proceso de aprendizaje. Está asociada a sentimientos negativos tales como el desasosiego, la frustración, la ansiedad, el miedo y la tensión. [...] Existen pocas disciplinas en el currículo, tal vez ninguna, que sean tan propensas a la ansiedad como el aprendizaje de lenguas extranjeras y de segundas lenguas. (Arnold y Brown, 1999: 263)

En conclusión, una enseñanza de idiomas que aspire a ser completa deberá incluir en el currículo tanto la dimensión afectiva del aprendizaje como la cognitiva, pues ambas se complementan. Los docentes de E/LE hemos tenido la oportunidad de comprobar en el aula la veracidad de esta afirmación en relación con nuestros alumnos de otros países y, muy en especial, con nuestros alumnos sinohablantes. 


\section{Desarrollo de la Unidad Didáctica}

\subsection{Estructura de la Unidad Didáctica}

El objetivo global de la unidad didáctica es producir una mejora en el ámbito del autoconocimiento y, por lo tanto, en la autoestima del estudiante. A través de la gestión de las propias emociones se busca una disminución de su impacto en el estado emocional del alumno, cuando estas sean negativas, y el desarrollo de herramientas afectivas que le permitan vincularse con emociones más positivas, puesto que es, en última instancia, en las emociones positivas en las que recae la capacidad de potenciar la adquisición lingüística. A «[...] la motivación, al fin y al cabo, se la orienta mejor dirigiéndola hacia el placer y hacia lo que Csikszentmihalyi (1990) llama flujo que alejándola del dolor» (Arnold y Brown, 1999: 257).

La unidad se divide en cinco actividades secuenciadas a partir de la combinación de tres parámetros estructurales:

1. Las competencias emocionales que el alumno debe fomentar para favorecer el buen desarrollo emocional.

2. Los pasos que hay que tener en cuenta para poder realizar un trabajo efectivo de reflexión sobre las propias emociones.

3. Las variables afectivas de la inteligencia intrapersonal orientadas al aumento de la autoconfianza.

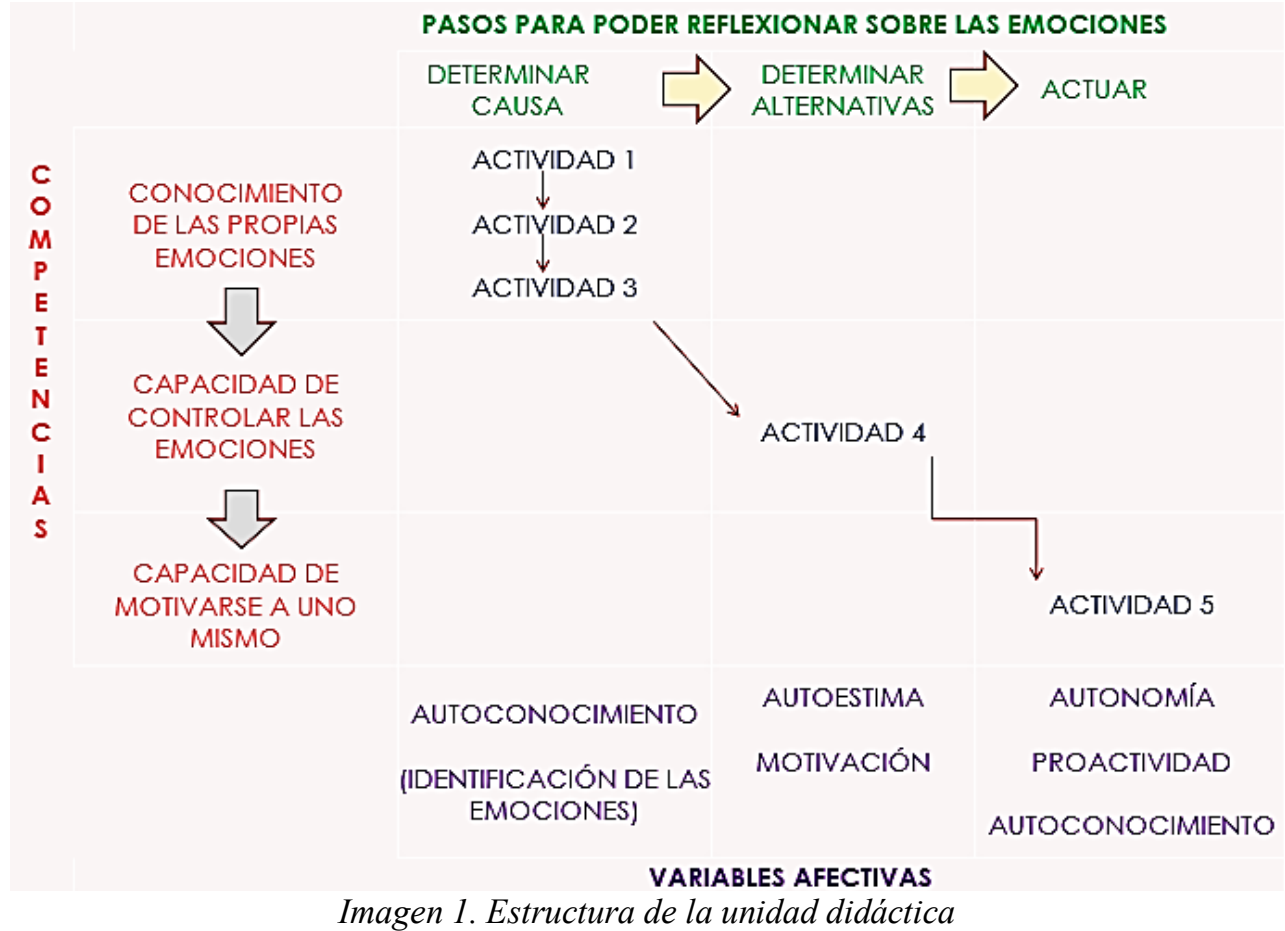

A la izquierda de la imagen se encuentra la columna vertebral que estructura la unidad didáctica. Se sigue la taxonomía de Mayer y Salovey (en Palmer et al. 2005: 286) para la inteligencia emocional. Mayer y Salovey conceptualizaron cinco competencias organizadas secuencialmente de las cuales las tres primeras se encargarían de estructurar la variable intrapersonal, mientras las dos últimas lo harían con la variable interpersonal. Estas tres competencias primeras son las que aquí se citan. La secuenciación, que se 
mueve del conocimiento emocional más básico al psicológicamente más complejo, quedaría así:

1. El conocimiento de las propias emociones: es la capacidad de reconocer un sentimiento en el mismo momento en que aparece. Representa la piedra angular de la inteligencia intrapersonal.

2. La capacidad de controlar las emociones: es la encargada de controlar las tensiones desagradables del ámbito emocional. Su carencia, por lo tanto, repercutirá en una incapacidad para modificar el propio estado de ánimo y, en consecuencia, el estudiante no podrá acceder a un estado emocional más adaptativo en relación con la situación de aprendizaje.

3. La capacidad de motivarse a uno mismo: es la variable que nos permite poder autocontrolarnos emocionalmente. Las personas que la posean podrán lograr más fácilmente resultados más sobresalientes a la hora de mantener la atención, la motivación y la creatividad, pues podrán acceder más fácilmente al estado de flujo ${ }^{1}$.

En la parte superior de la imagen están los tres pasos que se necesitan para poder realizar una reflexión efectiva de nuestras emociones. Hacer conscientes nuestros estados de ánimo y reflexionar sobre el antes, el durante y el después de la emoción hace que el flujo energético pueda entenderse, organizarse y, por lo tanto, usarse con consciencia para ser aprovechado a favor de la creatividad y de la acción efectiva. Estos pasos son:

1. Determinar la causa de la emoción: qué estado previo ha disparado esta energía en mí.

2. Buscar y determinar alternativas a esta emoción: ser consciente de que en última instancia yo soy responsable del estado emocional en el que me encuentro y, por lo tanto, capaz de orientar el flujo energético hacia emociones más constructivas y posibilitadoras.

3. Actuar: activar la capacidad de poner en marcha las acciones necesarias para provocar un cambio en la realidad que me rodea con el objetivo de cosechar los resultados deseados.

En la parte inferior de la imagen están las variables de la inteligencia intrapersonal que se trabajan en la unidad didáctica. Estas variables afectivas se secuencian en tres fases que intentan completar el ciclo del autoconocimiento:

1. Fase inicial: autoconocimiento.

2. Fase de desarrollo: desarrollo de la autoestima y de la motivación intrínseca.

3. Fase de consolidación: desarrollo de la autonomía y de la proactividad del estudiante y conocimiento consciente.

En lo que respecta a la tipología de las actividades, estas están pensadas para promover la autonomía del aprendiente y, también, para desarrollar la interacción grupal. Como forma de conseguir los objetivos enunciados, la unidad didáctica está compuesta por

\footnotetext{
${ }^{1}$ El estado de flujo es un estado de máximo rendimiento alcanzado durante la realización de una actividad en la cual se logra una alta productividad en relación con el esfuerzo invertido.
} 
actividades que combinan la interacción y la reflexión personal. A través de la interacción se pretende desarrollar las destrezas discursivas y afectivas de los estudiantes y mejorar su habilidad productiva en la L2. En esta misma línea, a través del fomento de la reflexión tiene lugar el trabajo de las variables de la inteligencia intrapersonal. Aunque se trabajan conjuntamente objetivos de carácter lingüístico y comunicativo, el foco de la unidad didáctica está puesto en el desarrollo de la variable afectiva.

\subsection{Ficha técnica}

\begin{tabular}{|l|l|}
\hline DESCRIPCIÓN DEL GRUPO META A & $\begin{array}{l}\text { Grupo monolingüe de alumnos } \\
\text { sinohablantes. } \\
\text { Nivel A2, B1. } \\
\text { Estudiantes universitarios con edades } \\
\text { comprendidas entre los 18 y 25 años. } \\
\text { Primer curso en inmersión. }\end{array}$ \\
\hline
\end{tabular}




\begin{tabular}{|c|c|}
\hline OBJETIVOS & 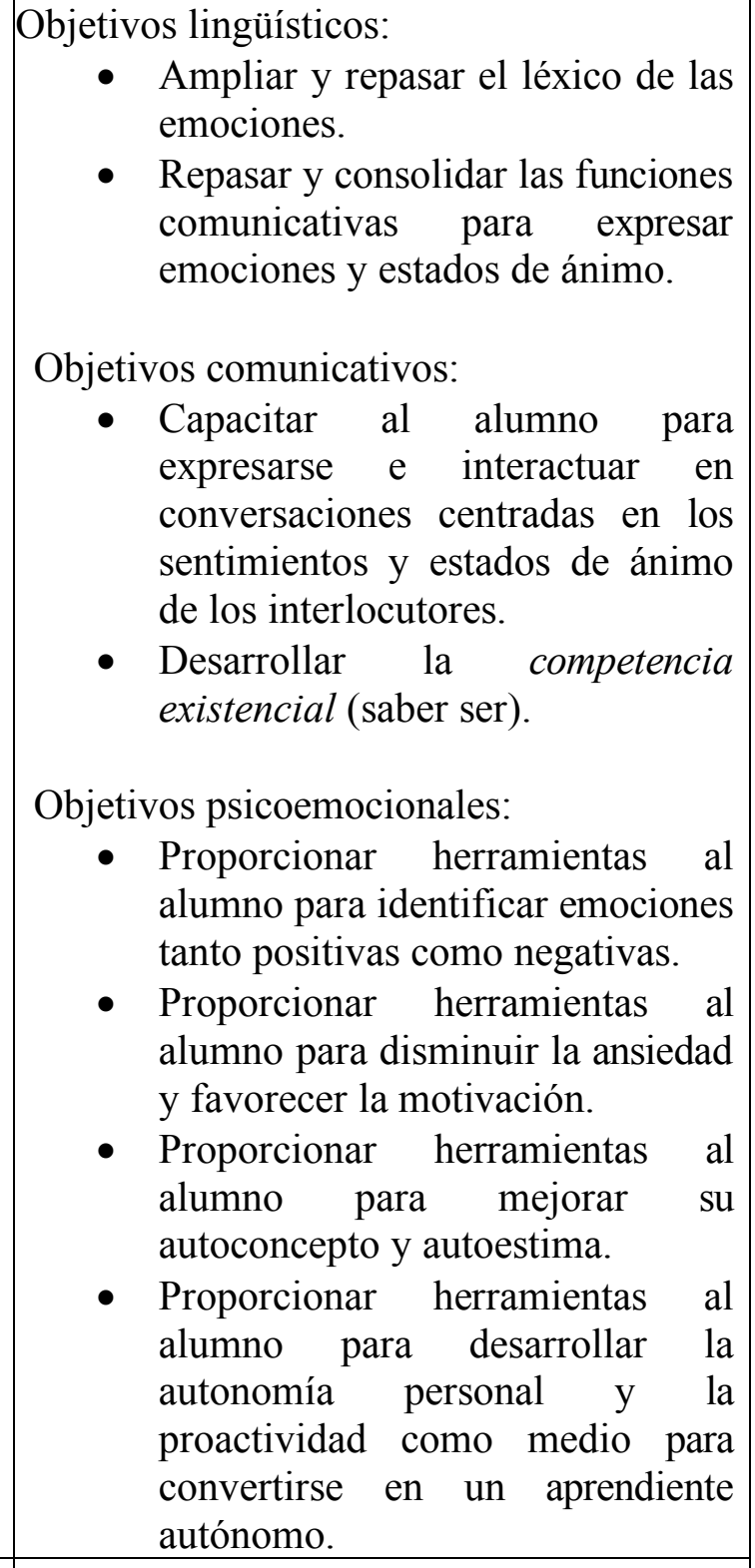 \\
\hline DESTREZAS & $\begin{array}{l}\text { Comprensión Oral, Comprensión Escrita, } \\
\text { Comprensión Auditiva, Expresión e } \\
\text { Interacción Oral, Expresión e Interacción } \\
\text { Escrita. }\end{array}$ \\
\hline CONTENIDOS & $\begin{array}{l}\text { Lingüísticos: } \\
\text { - Léxico de las emociones. } \\
\text { - Verbos de estado. } \\
\text { - Oraciones causales con porque }+ \\
\text { indicativo. } \\
\text { - Oraciones temporales con cuando } \\
+ \text { indicativo. }\end{array}$ \\
\hline
\end{tabular}




\begin{tabular}{|c|c|}
\hline & $\begin{array}{l}\text { - } \quad \text { Pretérito Indefinido. } \\
\text { - } \quad \text { Pretérito Imperfecto. }\end{array}$ \\
\hline DINÁMICA & Individual, grupo. \\
\hline DURACIÓN & 2 sesiones de 2 horas cada una. \\
\hline MATERIALES & $\begin{array}{l}\text { Pizarra, proyector, ordenador, altavoces, } \\
\text { láminas de libro Emocionario }{ }^{2} \text {. }\end{array}$ \\
\hline
\end{tabular}

Tabla 1. Ficha técnica de la unidad didáctica

\subsection{Explicación de las actividades en función de los objetivos psicoemocionales}

Esta unidad didáctica surge como intento de cubrir las necesidades afectivas de aprendizaje de los alumnos sinohablantes. Por esta razón, y a pesar de que los objetivos cognitivos de la unidad también son importantes, únicamente se van a desarrollar en este apartado los objetivos psicoemocionales.

Los objetivos psicoemocionales globales de la unidad didáctica (enunciados en la ficha técnica) buscan proporcionar herramientas al alumno para completar esta secuencia: detectar emociones negativas, disminuirlas, mejorarlas como medio de desarrollar la autoestima y el autoconcepto y, por último, desarrollar las habilidades necesarias para orientar al estudiante hacia una mayor proactividad.

En la actividad 1 se le pide al alumno que identifique siete emociones vinculadas con las variables de ansiedad, inhibición y autoestima. Los alumnos tendrán que leer las definiciones de las siete emociones y debatir entre ellos para adjudicar cada una de ellas a una lámina diferente. Estas emociones son: tensión, vergüenza, inseguridad, aburrimiento, entusiasmo, frustración y orgullo. Se trabajan a lo largo de toda la unidad didáctica con el objetivo de promover las mejoras buscadas en la autoestima del estudiante.

Esta actividad tiene por objetivo psicoemocional la definición de las emociones a partir de la práctica reflexiva con realia. Tomando como medio las definiciones y láminas del libro Emocionario se intenta que el alumno acceda a la emoción de forma consciente y sea capaz de nombrarla con exactitud. Si bien parece fácil, la denominación de las emociones más complejas puede ser un terreno resbaladizo.

${ }^{2}$ Emocionario es un libro publicado en España en el año 2013 en la editorial Palabras Aladas. Sus autores, Cristina Núñez Pereira y Rafael R. Valcárcel realizan un recorrido explicativo por una amplia gama de emociones humanas con el objetivo de servir de apoyo pedagógico para el desarrollo de la inteligencia emocional en los niños. A través de una serie de imágenes, en el libro se van desgranando los estados emocionales más habituales del ser humano y la relación que se establece entre ellos. Puede leerse eligiendo cada emoción al azar o siguiendo el recorrido emocional marcado por los autores. La premisa del libro es que el conocimiento de las propias emociones es la clave para la autoaceptación y un desarrollo psicoevolutivo sano. Puede encontrarse una descripción más detallada del libro en la página web de la editorial: https://www.palabrasaladas.com/ 
Por otro lado, una de las características de las emociones es que son innatas y automáticas, factor que juega en contra de la autoconsciencia. Aunque es cierto que, en líneas generales, todos somos capaces de reconocer las emociones, también es verdad que únicamente tenemos poder para transformarlas si somos conscientes de ellas en el momento exacto en el que las sentimos.

\section{$\underline{\text { Actividad 1 }}$}

A. Siempre estamos sintiendo emociones, pero ¿sabemos siempre cuál es la emoción que estamos sintiendo? A veces es dificil saberlo, por eso aquí tienes la definición de 7 emociones. Léelas y busca el vocabulario que no entiendas.

\begin{tabular}{|l|l|}
\hline La tensión: & Vocabulario nuevo que quiero recordar: \\
Cuando estoy tenso me siento nervioso, impaciente y & \\
estresado. Puedo sentirme tenso siempre que hago una & \\
cosa que me da miedo. Por ejemplo, cuando viajo a un país & \\
extranjero para vivir allí, cuando la profesora me pregunta & \\
en clase y tengo miedo de equivocarme o cuando discuto & \\
con un amigo. &
\end{tabular}

Imagen 2. Fragmento de la Actividad 1

D. Mira los dibujos, ¿cuál asocias a cada emoción? Primero piénsalo tú solo y, después, háblalo con tu compañero. Para ayudarte a encontrar la emoción de cada dibujo, aqui tienes algunas preguntas que puedes hacerte:

- ¿Qué colores hay en el dibujo?

- ¿Qué animales u objetos hay?

- ¿Qué ropa llevan?

- ¿Qué están haciendo los animales? ¿Se mueven? ¿Cómo se mueven? ¿Qué relación crees que hay entre ellos?

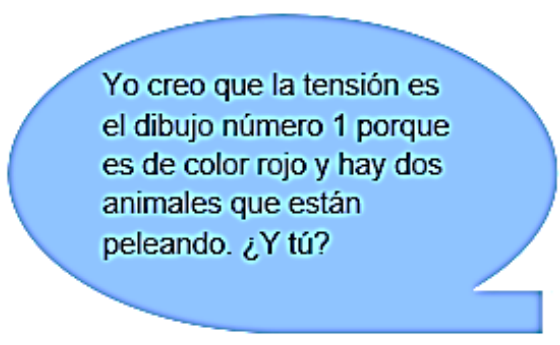

Pues yo creo que la vergüenza es el dibujo número...

\section{Imagen 3. Fragmento de la Actividad 1}

En la actividad 2 el alumno tiene que identificar las emociones que los protagonistas de la película de cine mudo El inmigrante, de Charles Chaplin, están sintiendo. La actividad se divide en dos ejercicios. En el primero se les pide que imaginen las emociones que los actores, en un barco rumbo al exilio a EE. UU., sentirán antes, durante y después del trayecto. En la segunda, los alumnos tienen que decidir cómo se sienten los protagonistas a partir de sus gestos en escenas concretas de la película. 
En la actividad 2 los objetivos psicoemocionales son identificar emociones en el otro a partir de la práctica reflexiva con realia e identificar y reflexionar sobre los propios estados emocionales. Con esta actividad se pretende que el alumno conecte de forma más estrecha con las emociones de la actividad 1, pero lo haga en un ambiente seguro. Para conseguir este objetivo, el visionado de una película puede ser un buen recurso. La identificación con el personaje suscita emociones en el alumno a través de las neuronas espejo.

E. Ahora vamos a ver fragmentos de la película, mientras lo hacemos fijate en los gestos de

los personajes. ¿Qué emoción crees que están sintiendo en cada momento? Puedes expresarlo

escribiendo o con mimica.

\begin{tabular}{|c|l|l|}
\hline MINUTO & \multicolumn{1}{|c|}{ EMOCIÓN } & \multicolumn{1}{c|}{$\begin{array}{c}\text { CAUSA Y LENGUAJE } \\
\text { CORPORAL }\end{array}$} \\
\hline $0: 55$ & $\begin{array}{l}\text { Charlot se siente: } \\
\text { A. orgulloso } \\
\text { B. frustrado } \\
\text { C. entusiasmado }\end{array}$ & $\begin{array}{l}\text { ¿Por qué? Porque ha pescado un } \\
\text { pez. } \\
\text { ¿Qué hace? Sonreir y mirar } \\
\text { mucho tiempo al pez. }\end{array}$ \\
\hline \multirow{2}{*}{$1: 47$} & $\begin{array}{l}\text { Ella está: } \\
\text { A. aburrida } \\
\text { B. triste } \\
\text { C. tensa }\end{array}$ & ¿Por qué? \\
& $\begin{array}{l}\text { Cuando Charlot y ella se ven, se sienten } \\
\text { A. entusiasmados } \\
\text { B. orgullosos } \\
\text { C. felices }\end{array}$ & ¿Por qué? \\
\hline $3: 50$ & ¿Qué hacen? \\
\hline
\end{tabular}

Imagen 4. Fragmento de la Actividad 2

En la actividad 3 el alumno tiene que escuchar las experiencias de aprendizaje de cuatro alumnos. Cada uno de ellos explica qué actividades y situaciones del aula les producen emociones positivas y negativas. En la segunda parte de la actividad nuestros estudiantes deberán completar una plantilla en la que reflexionen sobre dos emociones positivas y dos negativas que sientan en el aula con regularidad.

En la actividad 3 el objetivo psicoemocional es identificar y reflexionar sobre los propios estados emocionales en el aula de E/LE. Con esta actividad se pone en marcha el proceso de introspección personal. Una vez identificadas las emociones, se le pide al estudiante que sea capaz de enfrentarlas. Escuchar las experiencias de otros alumnos en el aula fortalece la empatía, al tiempo que se envía el mensaje de que sentir emociones negativas ante determinadas situaciones de aprendizaje es normal. Este hecho favorece la supresión de la soledad emocional que puede originarse al creer erróneamente que cada uno es el único que desarrolla emociones negativas en contextos de aprendizaje. Por último, tener que pensar en las ocasiones de su biografía en las que se han activado en él o ella esos sentimientos, ayuda al estudiante a poner en marcha el proceso de autoconocimiento interno. 
Actividad 3

A. Vas a escuchar a cuatro alumnos de español hablando de sus experiencias en el aula y de

cómo se sienten en algunos momentos. ¿Qué situaciones en el aula les hacen sentirse asi?

\begin{tabular}{|c|l|c|}
\hline ALUMNO & \multicolumn{1}{|c|}{ EMOCIÓN } & SITUACIÓN DEL AULA \\
\hline Yuki & emoción positiva: & \\
& emoción negativa: & \\
\hline Peter & emoción positiva: & \\
& emoción negativa: & \\
& & \\
& & \\
\hline
\end{tabular}

Imagen 5. Fragmento de la Actividad 3

En la actividad 4 los aprendientes han de leer un texto sobre la motivación y reflexionar sobre su capacidad para motivarse a sí mismos. Los objetivos psicoemocionales son:

1. Reflexionar sobre la importancia de conceptos clave para el buen desarrollo del proceso de adquisición lingüística de la L2: motivación, concepto de error, estrategias de aprendizaje.

2. Ayudar al alumno a regular las emociones limitadoras.

3. Ayudar al alumno a potenciar las emociones positivas.

4. Favorecer el desarrollo de la competencia de aprendizaje con el fin de potenciar las características del buen aprendiente de idiomas.

El objetivo último es, por lo tanto, hacer reflexionar al alumno sobre la necesidad de desarrollar la motivación intrínseca al ser esta la única que favorece la autonomía de aprendizaje y lo consolida en el largo plazo. Para esta actividad se ha elegido la Comprensión Lectora porque los conceptos a tratar son nucleares para el desarrollo de la identidad del alumno como aprendiente autónomo y, el texto, dado su carácter dialógico diferido, permite al estudiante adecuar la lectura a su ritmo de aprendizaje y volver a los contenidos siempre que lo desee. 


\section{$\underline{\text { Actividad } 4}$}

A. Aprender un idioma muevo es dificil, ¿verdad? Sobre todo, si es muy diferente de nuestro idioma, como lo son el chino y el español. Por eso es muy fácil sentir emociones negativas y perder la motivación, pero ¿es bueno para mí perderla? ¿Importa mucho o importa poco si mi motivación es poca? Vamos a leer un texto donde nos explican cómo funciona la motivación y cuánto la necesito. Léelo y responde a las preguntas:

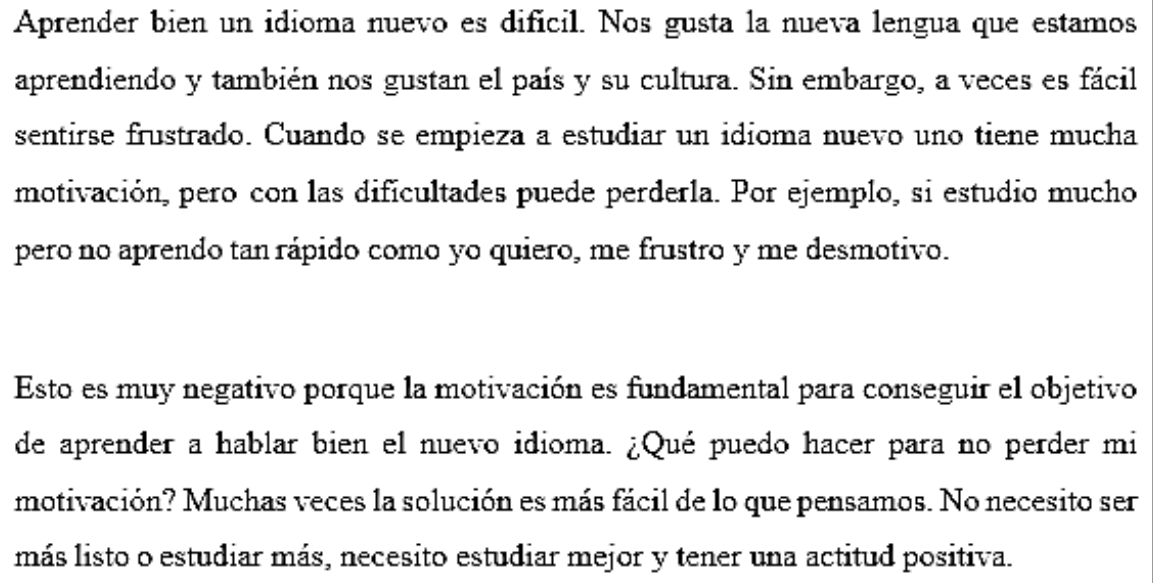

Imagen 6. Fragmento de la Actividad 4

En la actividad 5 el alumno tiene que completar la plantilla que rellenó en la actividad 3 con nueva información. Debe trabajar con las dos emociones negativas que ha anotado antes y asociarlas a una situación de su pasado en la que se sintió así y, a pesar de todo, logró completar su objetivo. También debe planificar la forma de superar la situación del aula que le produce las emociones negativas $y$, por último, anotar una frase motivadora.

El objetivo psicoemocional de esta actividad es que los alumnos aprendan la importancia que tienen para la motivación los mensajes que nos enviamos a nosotros mismos y tomen consciencia de que todos tenemos un diálogo interno susceptible de ser modificado en caso de que este nos dificulte la consecución de nuestras metas. Por último, se pretende conseguir que el estudiante dé el paso a la acción en la gestión de su propio proceso de aprendizaje. Para ello, se le anima a recapacitar sobre la importancia de fijar plazos y objetivos, así como de realizar compromisos y cumplirlos para conseguir las metas marcadas. 


\section{PLANTILLA DE EMOCIONES}

\begin{tabular}{|r|r|r|}
\hline me siento & cuando/ porque... & frase motivadora \\
\hline emoción positiva: & & \\
& & \\
& & \\
& & \\
& & \\
\hline
\end{tabular}

\begin{tabular}{|c|c|c|}
\hline me siento & cuando/ porque... & frase motivadora \\
\hline emociò negativa: & & \\
& & \\
& & \\
& & \\
& & \\
\hline
\end{tabular}

Imagen 7. Fragmento de la plantilla con la que concluye la Actividad 5 y la unidad didáctica.

\section{Conclusiones}

Dada su naturaleza, el trabajo de los componentes que constituyen la variable afectiva no se asemeja a los de la variable cognitiva. Al tratarse de elementos que conforman la identidad personal precisan de mayor duración en el tiempo. Es evidente que el trabajo sobre variables como la autoconfianza o la motivación no puede completarse en una unidad didáctica. Al contrario, su mejora, único objetivo factible, solo llegará tras un esfuerzo sostenido dentro y fuera del aula. Teniendo en cuenta esta consideración, la unidad didáctica que aquí se ha presentado no pretende ser sino una pequeña contribución al desarrollo de las habilidades intrapersonales de los alumnos.

Las conclusiones tras haber testado la unidad didáctica en el aula son fundamentalmente positivas. En términos generales, el grado de implicación de los alumnos fue alto. Como elementos mejor valorados por las ${ }^{3}$ docentes que ensayaron la unidad didáctica en el aula y sus alumnos encontramos el aprendizaje del vocabulario asociado a las emociones, la

\footnotetext{
${ }^{3}$ La unidad didáctica fue testada en dos grupos de alumnos sinohablantes de nivel A2 en situación de inmersión. Cada grupo constaba de quince alumnos y había sido formado hacía aproximadamente cuatro meses en el momento del ensayo de la unidad, por lo que el grado de confianza entre alumnos y docentes era alto.
} 
posibilidad de reflexionar sobre los propios estados emocionales (reflexión que en algún caso fue acompañada de una toma de consciencia sobre el sustrato emocional del alumno en el aula) y el trabajo colaborativo con el material de realia.

Estas valoraciones se encuentran en la línea de las conclusiones alcanzadas por Littlewood (en Azpiroz 2013: 43), quien afirma que los estudiantes chinos, en realidad, desearían participar más activamente en el aula. No se trata, por lo tanto, de que a estos estudiantes no les interese un aprendizaje que desarrolle el pensamiento crítico o el trabajo emocional, sino que carecen de las herramientas necesarias para llevarlo a cabo. Será labor de los profesores proporcionárselas y hacerlos sentir seguros y confiados durante el desarrollo de las actividades.

En primer lugar, la unidad sirvió para levantar del aula el tabú sobre las emociones. Desde el punto de vista del docente, la sensación que se tiene en muchas ocasiones en las aulas monolingües de alumnos sinohablantes es la de que hay una distancia de origen emocional que no puede ser nombrada. La unidad sirvió, por lo tanto, para romper esa prohibición. Por otro lado, tras su realización, algunas profesoras comprobaron que los alumnos mostraban un mejor reconocimiento de las propias actitudes en el aula y de su impacto en las emociones tanto propias como ajenas.

Es evidente, no obstante que, a pesar de los avances, unas variables cuyo origen se encuentra enterrado en los primeros años de vida del individuo sean difíciles de tratar en el aula de forma sistemática. Por lo tanto, la aplicación de la psicología a la pedagogía y la didáctica de segundas lenguas se hace necesaria para encontrar los caminos que permitan explorar todos los aspectos vinculados a la inteligencia intrapersonal con el objetivo de realizar propuestas didácticas significativas.

\section{Bibliografía}

AlCALÁ, F. (2002). «La Inteligencia Intrapersonal del alumno de idiomas». En M. C. Fonseca-Mora (ed.), Inteligencias múltiples, múltiples formas de enseñar inglés (pp. 115137). Sevilla: Mergablum.

ARnold, J. y H. D. Brown (2000). El aula de ELE: un espacio afectivo y efectivo. Recuperado el 09/01/20, de: http://cvc.cervantes.es/ensenanza/biblioteca_ele/publicaciones_centros/PDF/munich_20 05-2006/03_arnold.pdf.

AZPIROZ NúÑEZ, M C. (2013). «El estudiante chino de español como lengua extranjera (ELE). Diálogo entre la cultura china y el contexto educativo», Cuadernos de investigación educativa, 4, pp. 39-52. Recuperado el 09/01/20, de https://dialnet.unirioja.es/servlet/articulo?codigo $=5367397$.

Bega GónZALEZ, M. R. (2015). «Dificultades metodológicas de los estudiantes sinohablantes de español como lengua extranjera», Onomázein. Revista semestral de lingüística, filología y traducción, 32, pp. 227-238. Recuperado el 09/01/20, de: http://onomazein.letras.uc.cl/Articulos/N32/32_13_Bega.pdf.

CHIEN, Y. (2008). Cuestiones culturales y su relevancia en la enseñanza y el aprendizaje de español para los taiwaneses. Recuperado el 09/01/20, de: https://www.educacionyfp.gob.es/dam/jcr:631f5026-f55b-4fb2-a419-

024b4f53a3dc/2011-bv-13-32yi-shanchien-pdf.pdf. 
CSIKSZENTMIHALYI, M. (1996[1990]). Fluir. Una psicología de la felicidad (Nuria López, trad.). Barcelona: Editorial Kairós.

Couto Frías, M. S. (2014). «Alumnos chinos: ¿solos o acompañados?», SinoELE, 10, pp. 118- 126. Recuperado el 09/01/20, de: http://www.sinoele.org/images/Revista/10/Monograficos/EPES1011/EPES_I_118126.pdf.

Galloso CAMACHO, M. V. (2014): «Sistema de enseñanza en el aula de ELE en China», RAEL: Revista electrónica de lingüistica aplicada, 1, pp. 115-132. Recuperado el 09/01/20, de: https://dialnet.unirioja.es/servlet/articulo?codigo $=5031521$.

GARDNER, H. (1983). Frames of mind: The Theory of Múltiples Intelligences. New York: Basic Books.

GIBERT, M. I. y M. GuTIÉRREZ COLÓN (2014). «Estudiantes sinohablantes de español en programas de inmersión lingüística: ¿Fusión de metodologías?», Gramma, 53, pp. 92108 Recuperado el $09 / 01 / 20$, de: https://dialnet.unirioja.es/servlet/articulo?codigo $=6069286$.

GiovanNini, A. et al. (1996). Profesor en acción 1. Madrid: Edelsa.

GOLEMAN, D. (1997). Inteligencia emocional. Barcelona: Editorial Kairós.

LitTLEWOOD, W. (2000). «Do Asian students really want to listen and obey?», ELT Journal, 54 (1), pp. 31-36.

LU, J. (2009). «Métodos según las necesidades del alumnado: la enseñanza de ELE en China». En Y. Morimoto, M. ${ }^{a}$ V. Pavón y R. Santamaría (ed.), La enseñanza de ELE centrada en el alumno, (pp. 63-75). Asele.

PALMER., B. et al. (2005). «A psychometric evaluation of the Mayer-Salovey-Caruso Emotional Intelligence Test Version 2.0», Intelligence, 33, pp. 285-305.

SAlovey, P. y J. D. MAYER (1990). «Emotional intelligence», Imagination, Cognition and Personality, 9 (3), pp. 185-211.

SÁNCHEZ GRIÑÁN, A. (2009). «Estrategias de aprendizaje de ELE de sinohablantes (comprensión) y para sinohablantes (instrucción): un proyecto abierto», SinoELE, 1, pp. 1-12. Recuperado el 09/01/20, de: http://sinoele.org/images/Revista/1/estrategias.pdf.

VÁZQUez TORRONTERAS, A. (2018). «La identidad cultural en la R.P. China y su influencia en la enseñanza de ELE», redELE, 30, pp. 1-25. 